\title{
Effects of Wing-Cuff on NACA 23015 Aerodynamic Performances
}

\author{
S.M.A Meftah ${ }^{1}$, M. Belhenniche ${ }^{1}, \mathrm{O}_{\text {Madani Fouatih }}{ }^{1}$ and B. Imine ${ }^{\text {a }}$ \\ ${ }^{1}$ Laboratoire d'aéronautique et systèmes propulsifs, Département de Génie Mécanique Mechanical Engineering Faculty, \\ USTO Oran, Oran 31000 Algeria.
}

\begin{abstract}
The main subject of this work is the numerical study control of flow separation on a NACA 23015 airfoil by using wing cuff. This last is a leading edge modification done to the wing. The modification consists of a slight extension of the chord on the outboard section of the wings. Different numerical cases are considered for the baseline and modified airfoil NACA 23015 according at different angle of incidence. The turbulence is modeled by two equations k-epsilon model. The results of this numerical investigation showed several benefits of the wing cuff compared with a conventional airfoil and an agreement is observed between the experimental data and the present study. The most intriguing result of this research is the capability for wing cuff to perform short take-offs and landings.
\end{abstract}

\section{Introduction}

Since the publication of the first journals on the theory of boundary layer developed by Prandlt [1], a great challenge confronted scientists and engineers to minimize adverse effects and to further control flows. Different methods such as synthetic jets, vortex generators, vortex manipulator were frequently made to experience this problem with a varied degree of success. The need for flow control emerged from the sixties years primarily to reduce wall friction, delay separation and reduce noise and vibration. To control the detachment of boundary layer, it became natural to intervene in the flow near the wall; because it is acting at the boundary layer it is possible to influence the aerodynamic characteristics of a global airfoil. Originally, the means of control were passive; they do not require an external energy supply. With the development of new technologies and improved knowledge about the internal dynamics of the turbulent boundary layer, control techniques available, passive or active, were extremely diverse. Since the publication of the first journals on the theory of boundary layer developed by Prandlt[1], a great challenge confronted scientists and engineers to minimize adverse effects and to further control flows.

Different methods such as synthetic jets, vortex generators, vortex manipulator were frequently made to experience this problem with a varied degree of success. A vast literature accumulated over the years in this area was actually reviewed by several authors as Ostowari [2], Tani [3], Chang [4] and others. However, the use of movable walls to control the boundary layer has received relatively less important. This technic called moving surface boundary-layer control is an active actuator to delay separation and control the flow of fluid around the body and improve the aerodynamic characteristics such as lift and drag. However, very few studies have focused on the complete characterization of the numerical and experimental mechanism and its influence on the flow. Similarly, the problems associated with the use of actuators have been little discussed. Among the most effective mechanisms for control by moving surface, we have to include the airfoils with wing cuff at the leading edge which is the subject of our present study. In 1983, the "NASA Langley Research Center" [5] presented a study on the increased resistance to rotation on a general aviation aircraft. Adding the wing cuff creates a secondary vortex delaying a fall on the diffusion of the wing. For high angles of incidence, where a large part of the wing is lifted, the flow on the outer part of the wing is attached to the form. This study found that a device leading edge can increase the critical angle stall more than $20^{\circ}$ degrees and increase control during phases in small roll thereby improving lateral stability. The study showed that virtually increases the coefficient of the resultant force was mainly due to the increase in lift more than the drag force.

In the present work, a numerical investigation of flow around a modified NACA 23015 is presented. As shown in Figure 1, the modified airfoil is the cord $\mathrm{c}=150 \mathrm{~mm}$ has an extension of the leading edge applied to a portion $37 \%$ of the wing spans. For all tested cases, a Reynolds number of $2.31 \times 10^{5}$ was considered and equivalent to inlet velocity of $20 \mathrm{~m} / \mathrm{s}$ according at different angle of incidence between $0^{\circ}$ to $36^{\circ}$. The present study will be

\footnotetext{
a Corresponding author: imine_b@yahoo.fr
} 
applied in the boundary layer which is the genesis of the phenomena governing the global dynamics of such flow control and aerodynamic performance of the modified airfoil.

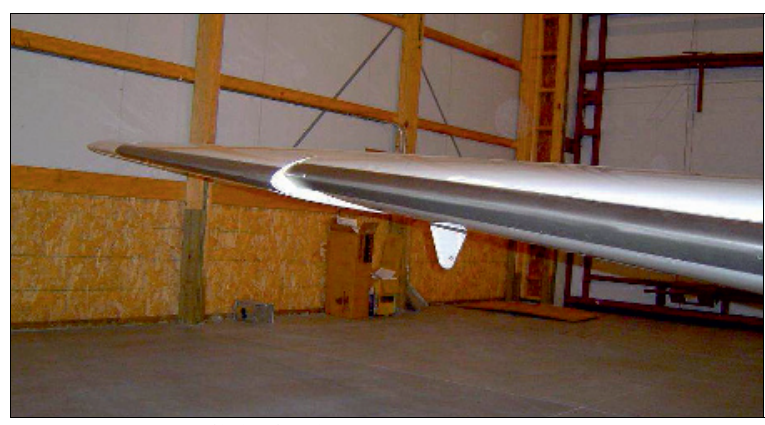

Figure 1. Modified wing

\section{Transport equations and turbulence model}

The flow around the airfoil is considered turbulent asymmetrical. The general forms of the transport equations can be written in Cartesian coordinates as:

Continuity equation

$$
\frac{\partial}{\partial x_{j}}\left(\bar{\rho}_{U_{j}}\right)=0
$$

Momentum conservation equation

$$
\begin{aligned}
& \frac{\partial}{\partial x_{j}}\left(\bar{\rho} \widetilde{U}_{i} \widetilde{U}_{j}\right)=\bar{\rho} g_{i}-\frac{\partial \bar{p}}{\partial x_{i}}-\frac{\partial}{\partial x_{j}}\left(\rho_{u_{i}}{ }^{\prime \prime} u_{j}{ }^{\prime \prime}\right) \\
& +\frac{\partial}{\partial x_{j}}\left[\bar{\mu}\left(\frac{\partial \widetilde{U}_{i}}{\partial x_{j}}+\frac{\partial \widetilde{U}_{j}}{\partial x_{i}}\right)-\frac{2}{3} \bar{\mu} \frac{\partial \widetilde{U}_{k}}{\partial x_{k}} \delta_{i j}\right]
\end{aligned}
$$

The model of turbulence used in this present work is a model with two transport equations k-epsilon:

$$
\begin{gathered}
\frac{\partial}{\partial t}(\bar{\rho} \tilde{k})+\frac{\partial}{\partial x_{i}}\left(\bar{\rho} \widetilde{k} \widetilde{u}_{i}\right)=\frac{\partial}{\partial x_{j}}\left[\left(\mu+\frac{\mu_{t}}{\sigma_{k}}\right) \frac{\partial \widetilde{k}}{\partial x_{j}}\right]+G_{k}+G_{b}-\rho \mathcal{\varepsilon}-Y_{M}+S_{k} \\
\frac{\partial}{\partial t}(\rho \widetilde{\varepsilon})+\frac{\partial}{\partial x_{i}}\left(\rho \widetilde{\tilde{d}_{i}}\right)=\frac{\partial}{\partial x_{j}}\left[\left(\mu+\frac{\mu_{i}}{\sigma_{k}}\right) \frac{\partial \widetilde{\varepsilon}}{\partial x_{j}}\right]+ \\
C_{1 \varepsilon} \frac{\widetilde{\widetilde{\varepsilon}}}{\widetilde{k}}\left(G_{k}+C_{3 \varepsilon} G_{b}\right)-C_{2 \varepsilon} \rho \frac{\widetilde{\varepsilon}^{2}}{\widetilde{k}}+S_{\varepsilon}
\end{gathered}
$$

Production of turbulent kinetic energy $G_{k}$ is given by the formula:

$$
G_{k}=-\bar{\rho} \cdot \overline{u_{i}^{\prime \prime} \cdot u_{j}^{\prime \prime}} \frac{\partial \tilde{u}_{j}}{\partial x_{i}}
$$

The turbulent viscosity is obtained by:

$$
\mu_{t}=\bar{\rho} C_{\mu} \frac{\widetilde{k}^{2}}{\widetilde{\varepsilon}}
$$

The model constants are given in Table 1:

Table1. The model constants

\begin{tabular}{|c|c|c|c|c|}
\hline$C_{1 \varepsilon}$ & $C_{2 \varepsilon}$ & $C_{\mu}$ & $\sigma_{k}$ & $\sigma_{\varepsilon}$ \\
\hline 1,44 & 1,92 & 0,09 & 1,0 & 1,3 \\
\hline
\end{tabular}

(For more information see reference 3)

\section{Meshing and boundary conditions}

For the airfoil case, NACA 23015 airfoil with $0.150 \mathrm{~m}$ of chord length $c$ and as it shown in figures 2 and 3, the 3D structured mesh are generated. The upstream boundary of the computational domain is $3 c$ from the leading edge of the airfoil. The outflow boundary is located at $12 c$ downstream of the trailing edge. Steady threedimensional, incompressible flow is solved. Turbulence is modeled by two equations $\mathrm{k}$-epsilon model. Inlet boundary conditions are corresponding to the boundary conditions of the experiment [5]. Second order upwind discretization scheme is selected with respect to the mesh used. The simulation is run acquired at $0^{\circ}$ through $36^{\circ}$ of incidence angle where stalling occurred. The Reynolds number based on the free stream velocity and the chord length is $2.31 \times 10^{5}$. To achieve this Reynolds number, the velocity inlet is set at $20 \mathrm{~m} / \mathrm{s}$.

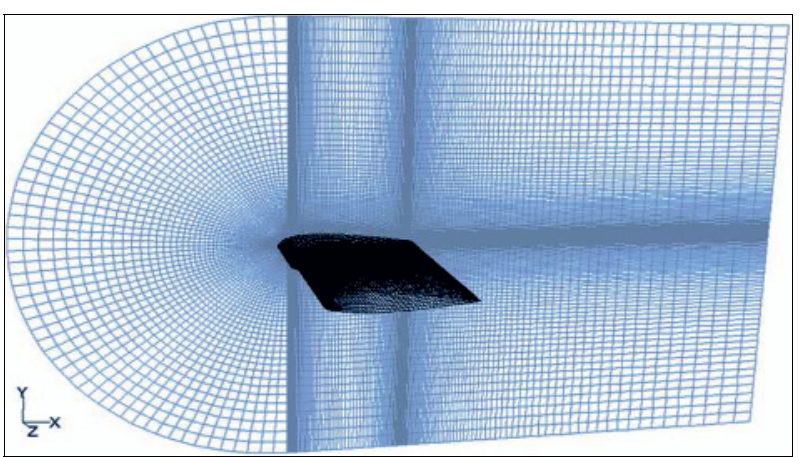

Figure 2. Meshing of calculation domain

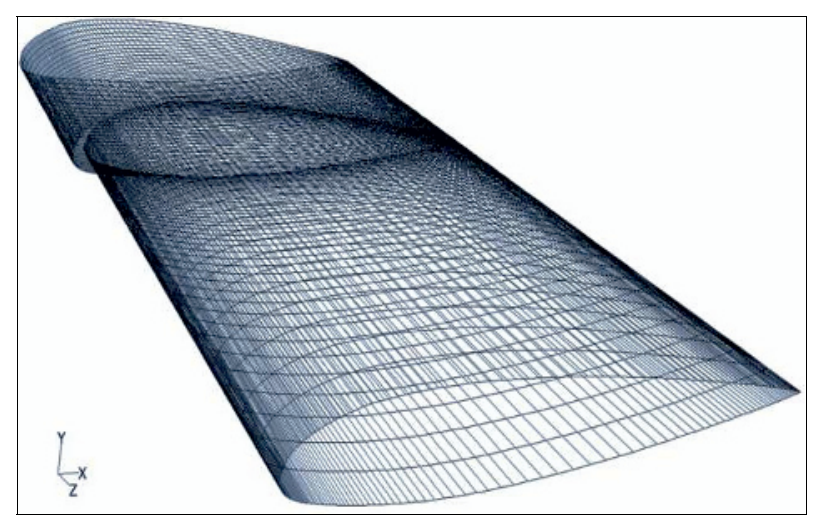

Figure 3. Meshing of airfoil with cuff 


\section{Results and discussion}

Several compilations were carried out for different angles of incidence to determine the lift and drag. Each case was carried out at the same velocity: 20 meters per second. The angle of incidence extended from 0 to 30 degrees.

In CFD modeling, the solution should be independent of the mesh density to ensure the realism of the solution gives the solver after convergence. The independence of the simulation results with respect to the mesh was confirmed after a series of tests on several mesh densities (see table 2).

Table2. Meshing

\begin{tabular}{|c|c|}
\hline Meshing & $\begin{array}{l}\text { Number of } \\
\text { cells }\end{array}$ \\
\hline 1 & 617.310 \\
\hline 2 & 924370 \\
\hline 3 & 1232630 \\
\hline 4 & 1542090 \\
\hline 5 & 1858560 \\
\hline
\end{tabular}

Figure 4 and Figure 5 show respectively the variation of the pressure coefficient and velocity magnitude depending on the position with respect to the rectilinear axis of abscissas of the computational domain. We note that the values of the variables presented two latest meshes are superimposed on each other which mean that beyond accuracy applied to mesh Number 4 (see table.2), the solution becomes independent of the resolution and quality of the mesh. This means that the parameters of the solution become numb from the higher number of stitches or equal approximately 1,542,090 and is the mesh that has been adopted.

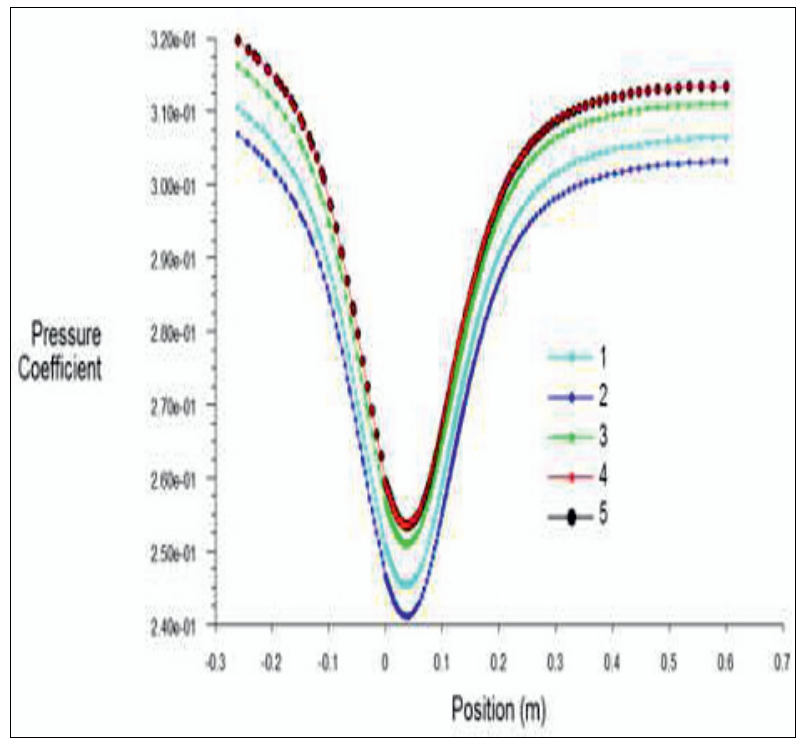

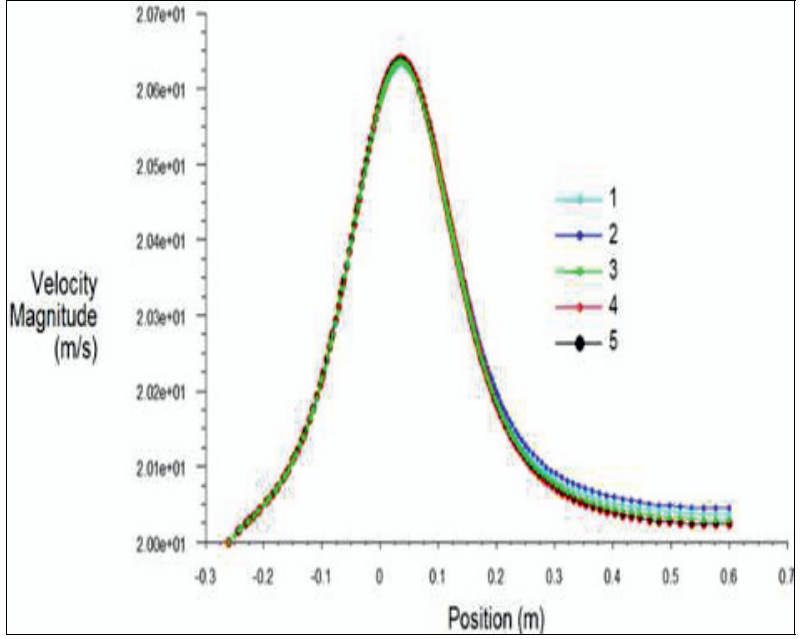

Fig.5. velocity magnitude

By observing Figure 6, we could see the difference between the modified wing and the unmodified one. It is obvious that there is little or no difference between the two configurations at $0^{\circ}$ of incidence. The flow is accelerated in the near-wall areas, large pressure gradients at the leading edge of the profile where a slowing of the flow is observed. Near the angle of 16 degrees, the flow over the wing is largely separated from unmodified wing. However, at the same angle of attack, the modified over the outer wing section is attached. The modified wing always shows the disturbed flow over the wing section of the unmodified, but over the news flow is regular.
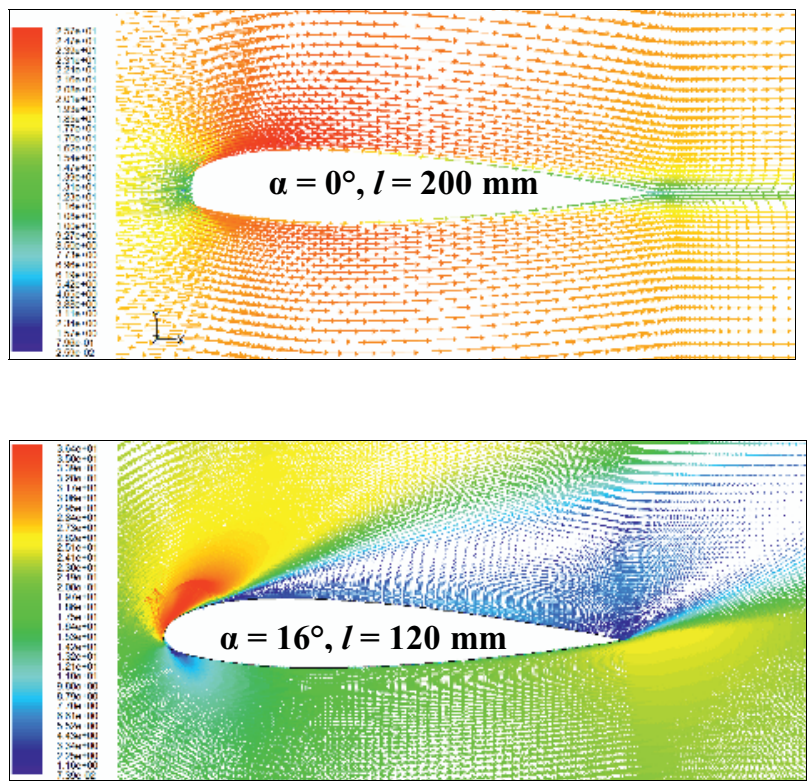

a. Unmodified airfoil

Figure 4. Pressure coefficient 

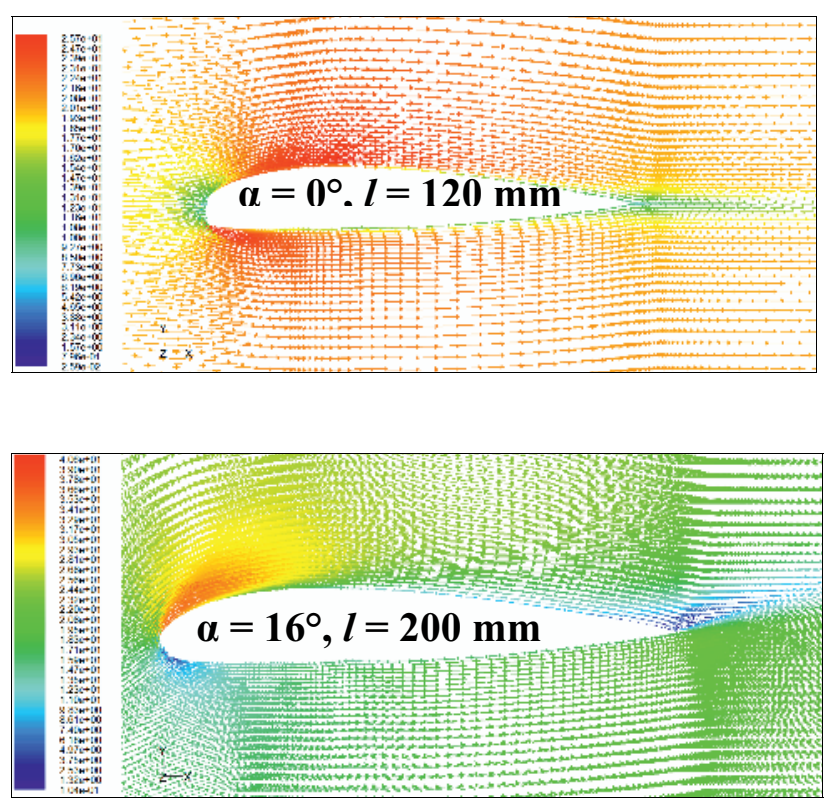

b. Modified airfoil

Figure 6. Computational visualizations of field vectors

In the figure 7 , the curve of lift coefficient confirms quantitatively delaying the separation of the boundary layer, including significant gains in lift. Our mathematical model used in this configuration gave us a very good approximation for values obtained by comparing them with the experimental results reported in the literature [5]. However, the control effect of the separation is significant on the aerodynamic coefficients. Also, the figure 7 shows the gains obtained with the lift installation of the Wing-Cuff into the leading edge. In their presence, the lift increases significantly in the case of profile with cuff, indicating that the dropout occurs around $32^{\circ}$ angle with an increase in the lift coefficient of about $32 \%$. The addition of the cuff edge created a secondary vortex which delayed the spread of drop above the wing in the spanwise. At high angles of incidence, where a large part of the wing is lifted, the flow over the outer section of the wing and therefore above the cuff is attached to the profile.

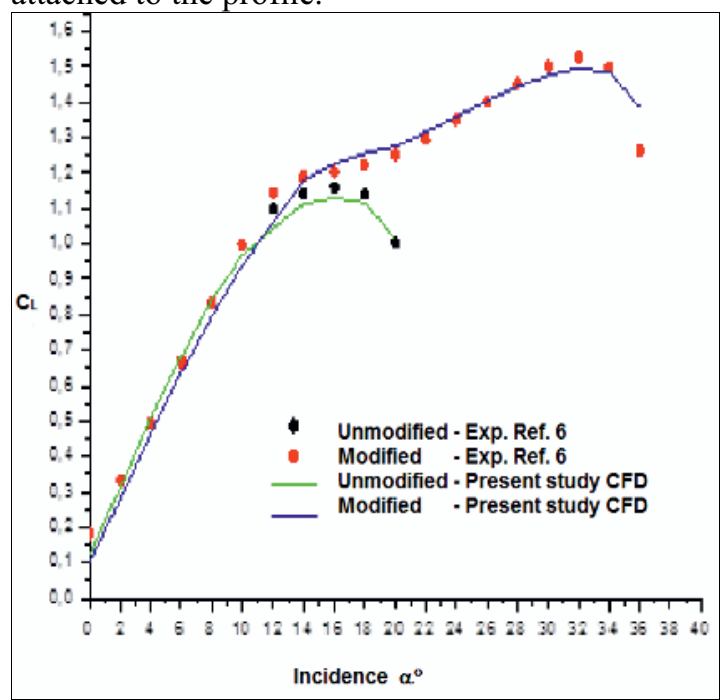

Figure 7. Evolution of the lift coefficient
The figure 8 shows the evaluation of the coefficient of drag depending on the angle of incidence of the flow. From Figure 5.13, we note that the change that was made by the location of the cuff edge has affected the variation of the drag coefficient slightly what appears logical because the shape of the edge incidence has not been changed on a major part of the profile $(37 \%)$.

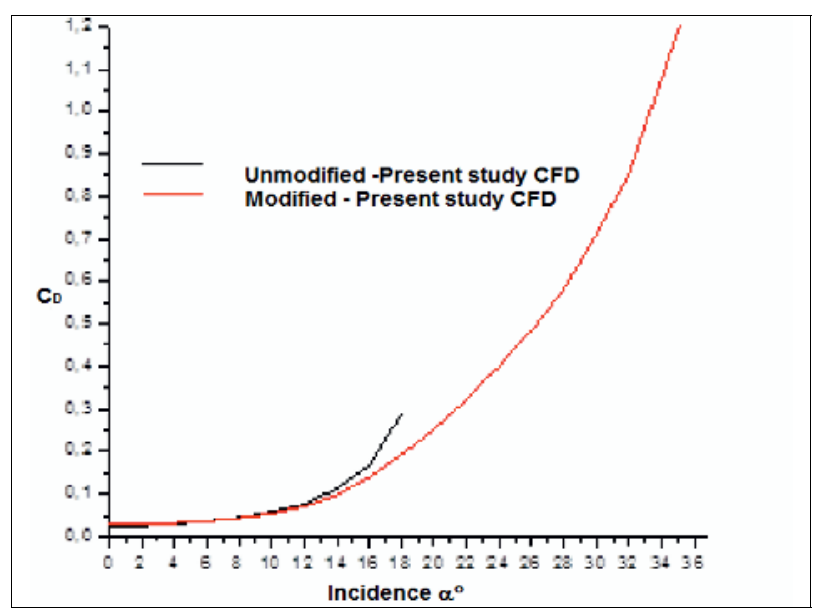

Figure 8. Evolution of the drag coefficient

\section{Conclusions}

The results of this numerical investigation showed several benefits of the wing-cuff compared with a conventional airfoil. Also, the results showed that the addition of the outboard leading-edge droop eliminated the abrupt stall of the wingtip and maintained or increased the resultant-force coefficient up to about 32 degrees. This change in slope of the resultant-force coefficient curve with angle of attack from at higher angles of attack has been shown to be important to perform short take-offs and landings.

\section{References}

1. S.H. Lam, The Prandtl Boundary Layer Theory. ME351B Fluid Mechanics, Stanford University (Win) 2003-04.

2. C. Ostowari, D. Naik, Post stall studies of untwisted varying aspect ratio blades with an NACA4415 airfoil section-part 1. Wind Engineering 1984; 8 (3): 176-194.

3. I. Tani, Boundary-Layer Transition, Annual Review of Fluid Mechanics 1: 169-196

4. R.D. Joslin, C.L. Streett, I. Chang, Spatial Direct Numerical Simulation of Boundary-Layer Transition Mecha-nisms: Validation of PSE Theory Theor. \& Comput. Fluid Dyn, 1993.

5. H. P. Stough, D.J. DiCarlo, E.C. Stewart, "Wing Modification for Increased Spin Resistance", NASA Langley Research Center, SAE Technical Paper Series, Paper 830720, Business Aircraft Meeting \& Exposition, Avril 1983 